\title{
Comorbidity and treatment issues in juvenile bipolar disorder Giulio Perugi
}

\author{
Address: Istituto di Scienze del Comportamento G De Lisio, Clinica Psichiatrica Università di Pisa, Italy \\ from International Society on Brain and Behaviour: 3rd International Congress on Brain and Behaviour \\ Thessaloniki, Greece. 28 November - 2 December 2007 \\ Published: 17 April 2008 \\ Annals of General Psychiatry 2008, 7(Suppl I):S43 doi:I0.I I86/I744-859X-7-SI-S43
}

This abstract is available from: http://www.annals-general-psychiatry.com/content/7/SI/S43

(c) 2008 Perugi; licensee BioMed Central Ltd.

Juvenile bipolar disorder (BD) is reported to be more treatment-resistant than adult $\mathrm{BD}$, and to show poorer outcome. Predictors of treatment non-response in early onset BD are not well defined. Many potential domains of variables may be considered. Age, gender and age at onset of BD can affect both clinical presentation and pattern of comorbidity, and both these issues may affect the pharmacological response. Some features, which in adult patients with $\mathrm{BD}$ are considered predictors of poor treatment response, such as severity, mixed states, psychotic symptoms, and co-morbid substance abuse are particularly frequent in youths, and they also may influence pharmacological response in children and adolescents as well. We explored this issue in a study, conducted in the last 3 years, aimed to explore the role of lifetime comorbid externalizing and internalizing disorders as possible predictors of treatment non-response, in bipolar children and adolescents with manic or mixed episodes. Nonresponders had more frequently co-morbid conduct disorder and/or ADHD. Furthermore, they were globally more severe at baseline and required more frequent addition of antipsychotic medications than treatmentresponder patients. Co-morbid anxiety disorders in our sample did not predict treatment resistance. Interestingly, anxiety disorders and $\mathrm{CD}$ were inversely related, with the number of anxiety disorders significantly lower in subjects without CD comorbidity. The use of antipsychotics was associated with treatment non-response, and it was probably related to the severity of the subjects who received these drugs (i.e. higher prevalence of impulsivity, psychomotor agitation, behavioral problems, psychotic symptoms). Different mechanisms can be involved in treatment-resistance of bipolar subjects with co-morbid externalizing disorders. BD plus externalizing disorders may represent a specific subtype, with earlier-onset and resistance to traditional anti-manic and mood stabilizing drugs. Poorer treatment response in BD with co-occurring externalizing disorders may be also accounted for by a more problematic compliance to treatment. Several comorbidities with negative prognostic implications (i.e. substance abuse, antisocial behaviors), which are more frequent in bipolar patients with externalizing disorders, may further affect clinical course. Follow-up studies with larger samples of subjects with BD and/or externalizing disorders appropriately treated with different options are needed to address this important issue. Additional stimulant medication after adequate coverage with mood stabilizers might represent one rational approach to this group of refractory bipolar children. 